\title{
Variation of Peripheral Th17/Treg Imbalance in Patients with Idiopathic Membranous Nephropathy After Cyclosporin a Treatment: A Prognostic Marker of Idiopathic Membranous Nephropathy
}

\author{
Dan Tang ${ }^{1}$, JiGuang Guo ${ }^{1}$ and JianBin Zhang* \\ Department of Nephrological Diseases, Affiliated Yong Chuan Hospital of Chong Qing Medical University, China
}

Received: 阱 July 30, 2018; Published: 盋 August 13, 2018

*Corresponding author: JianBin Zhang, Department of Nephrological Diseases, Affiliated Yong Chuan Hospital of ChongQing Medical University, China

\begin{abstract}
Objective: To investigate the variation and effect of peripheral Th17 and regulatory T (Treg) cells upon the clinical prognosis of idiopathic membranous nephropathy (IMN) patients after cyclosporin A (CsA) treatment.

Methods: Twenty-four IMN patients with 24-hour urinary protein level $\geqq 4 \mathrm{~g}$ were recruited. According to 24-hour urinary protein levels, all patients were assigned into the high-and middle-risk groups. All enrolled patients received standardized treatment of prednisolone acetate in combination with CsA. The Treg and Th17 frequencies were measured by flow cytometry. Serum cytokines were detected by enzyme-linked immunosorbent assay. Serum albumin and 24-hour urinary protein were measured by fully automatic biochemical analyzer.

Results: Compared with the healthy controls, Th17\%, levels of IL-17 and TNF- $\alpha$ were up-regulated, whereas Treg\% and TGF- $\beta$ level were down-regulated in the peripheral blood of IMN patients. This imbalance was more pronounced in the high-risk group compared with middle-risk groups. The 24-hour urinary protein level was positively correlated with Th17\% and Th17/Treg ratio, whereas negatively correlated with Treg\%. After 6-month treatment, Peripheral Th17 frequency, IL-17 and TNF- $\alpha$ level decreased, whereas Treg frequency and TGF- $\beta$ level increased in the effective group (all $\mathrm{P}<0.05$ ). No significant changes were found in the ineffective group. Conclusion: IMN patients present with peripheral Th17/ Treg imbalance, which is correlated with the severity of IMN. CsA treatment is an effective approach to improve peripheral Th17/Treg imbalance in IMN patients, which is associated with the clinical efficacy of CsA treatment. Monitoring the variations in peripheral frequency of Treg and Th17 is of significance for evaluation of the severity of IMN and clinical efficacy.
\end{abstract}

Keywords: Th17; Treg; Idiopathic membranous nephropathy; Cyclosporin A; Efficacy evaluation

\section{Introduction}

Idiopathic membranous nephropathy (IMN) is a common cause of nephrotic syndrome in adults. Previous investigations at home and abroad reported that IMN accounts for approximately $1 / 3$ of nephrotic syndrome with the highest percentage [1]. After immune therapy, partial IMN patients obtain no evident remission and eventually progress into end-stage renal disease. The exact pathogenesis of IMN remains to be fully elucidated by detection parameters with high sensitivity and specificity. Recent investigations have demonstrated that the peripheral levels of ICOS (+) and PD-1 (+) Tfh cells are significantly up-regulated in IMN patients, which can be utilized to predict the development and progression of IMN [2], prompting that cellular immunity disturbance probably plays a vital role in the pathogenesis of IMN. In recent years, the Th17/ Treg balance, which is different from CD4+ T-helper lymphocyte subset of Th1 and Th2, has been identified to play an influential role in the regulation of host immune tolerance, resistance of rejection reaction, infection, malignant tumor, inflammation and alternative diseases [3].

Multiple studies [4-5] have suggested that Th17 and its primary secretion IL-17 are involved with the pathogenesis of lupus nephritis, crescentic glomerulonephritis and proliferative glomerulonephritis, etc. Previous investigations have demonstrated that the peripheral Treg\% in IMN patients is significantly lower compared with that in the healthy controls. After rituximab monoclonal antibody therapy, peripheral Treg\% is elevated, which is intimately correlated with clinical efficacy [6]. Nevertheless, whether Th17/Treg imbalance exists in IMN patients and its correlation with the progression and clinical prognosis of IMN patients has been rarely reported. Cyclosporin A (CsA) is a highly selective potent immunosuppressive agent, which can effectively 
inhibit the proliferation and activation of $\mathrm{T}$ lymphocytes. Current studies $[7,8]$ have reported that use of CsA exerts significant effect upon the Th17/Treg ratio in patients with autoimmune diseases and after organ transplantation. In addition, the variation in Th17/ Treg ratio is probably correlated with clinical efficacy. However, the effect of CsA therapy upon the changes in Th17/Treg ratio in IMN patients has been seldom reported. Consequently, this study was designed to investigate the changes in Th17/Treg ratio before and after CsA therapy and its correlation with clinical prognosis of IMN patients.

\section{Materials and methods}

\section{Baseline Data}

Twenty-four IMN patients with 24-hour urinary protein level $\geqq 4 \mathrm{~g}$ admitted to our hospital between September 2015 and October 2016 were recruited in this clinical trial. The mean age of the patients was $48.83 \pm 4.92$ years (range 20-77 years), 15 male and 9 females. All patients were pathologically diagnosed with membranous nephropathy via renal biopsy. The possibility of alternative secondary membranous nephropathy was excluded. All enrolled patients were administered with hormone $(0.5 \mathrm{mg} /$ $\mathrm{kg}^{*} \mathrm{~d}$ ) in combination with CsA (3-5 mg/kg*d). The blood drug concentration of CsA was monitored and maintained to the standard range of 100-180 ng/ml. According to the KDIGO guideline approach, all patients were divided into the high- and middlerisk groups according to quantitative analysis of 24-hour urinary protein. In the middle-risk group, the renal function was normal, and the albuminuria level ranged from $4 \mathrm{~g} / 24 \mathrm{~h}$ to $8 \mathrm{~g} / 24 \mathrm{~h}$. In the high-risk group, renal insufficiency or kidney atrophy was noted, and the albuminuria level exceeded $8 \mathrm{~g} / 24 \mathrm{~h}$. Quantitative analysis of 24-hour urinary protein was utilized as a parameter to evaluate the clinical efficacy.

Inclusion criteria were as follows:

a) patients of both genders aged $\geq 18$ years;

b) those pathologically diagnosed with membranous nephropathy via renal biopsy;

c) those diagnosed with IMN for the first time and had no medical history of cyclophosphamide,Cys A or immunosuppressive agent use.

Exclusion criteria were as below:

a) patients complicated with infection, malignant tumor, hypertension and diabetes mellitus, etc.;

b) pregnant women. Twelve age- and gender-matched healthy controls, 7 male and 5 females were recruited in this clinical trial.

All enrolled individuals had no short-term history of immune or infectious diseases. All patients had signed the informed consents and completely cooperated with the study procedures.

\section{Methods}

A portion of 8-20 $\mathrm{ml}$ of fasting venous blood samples were collected from both the IMN patients and healthy controls before and 6 months after corresponding treatment. The plasma was separated from the cells within $2 \mathrm{~h}$ after blood sampling collection. The serum levels of ALB and 24-hour urinary protein were detected by fully automatic biochemical analyzer. The expression levels of IL17 , TNF- $\alpha$ and TGF- $\beta$ were quantitatively measured by ELISA. The serum Th17\% and Treg\% were detected and calculated by flow cytometry.

\section{Statistical Analysis}

SPSS 20.0 software package was utilized for statistical analysis. Measurement data were expressed as mean \pm standard deviation and analyzed by using t-test. Enumeration data were expressed in percentage and statistically processed by chi-square test. A P value of less than 0.05 was considered as statistical significance.

\section{Results}

\section{Baseline Data}

Baseline data of the IMN patients and healthy controls were illustrated in Table 1. No statistical significance was identified in gender, age and renal function between two groups (both $\mathrm{P}>0.05$ ). However, quantitative test of 24-hour urinary protein was higher while serum albumin levels was lower in IMN patients (both $\mathrm{P}<0.05$ ).

Table 1: Baseline data of the enrolled study subjects.

\begin{tabular}{|c|c|c|}
\hline & $\begin{array}{c}\text { IMN patients } \\
(\mathbf{n = 2 4 )}\end{array}$ & $\begin{array}{c}\text { healthy control } \\
(\mathbf{n = 1 2 )}\end{array}$ \\
\hline Sex (male: female) & $15 / 9$ & $7 / 5$ \\
\hline Age (year) & $48.21 \pm 8.69$ & $48.55 \pm 5.82$ \\
\hline 24-hour urinary protein (g) & $6.82 \pm 3.89 *$ & $0.10 \pm 0.02$ \\
\hline Serum ALB levels (g/L) & $25.44 \pm 4.91^{*}$ & $40.55 \pm 3.21$ \\
\hline $\begin{array}{c}\text { Creatinine clearance (ml/ } \\
\text { min) }\end{array}$ & $91.50 \pm 4.13$ & $97.70 \pm 3.82$ \\
\hline \multirow{2}{|c|}{$* \mathrm{P}<0.05$ vs. heathy control }
\end{tabular}

\section{Leukocyte Counts and Cytokine Concentration in IMN Patients}

Compared to healthy group, IMN group exhibited higher Th17\% (1.15 $\pm 0.62 \%$ vs. $0.77 \pm 0.22 \%, \mathrm{P}<0.05$ ), and lower Treg\% $(0.96 \pm 0.59 \%$ vs. $1.67 \pm 0.52 \%, \mathrm{P}<0.05) \quad$ (Table 2). The related cytokine concentrations IL-17and TNF-a in IMN group is higher $(56.02 \pm 9.54$ vs. $43.6 \pm 8.81$ and $149.8 \pm 13.09$ vs. $36.51 \pm 7.79$, $\mathrm{P}<0.05)$ while TGF- $\beta$ is lower than healthy group $(678.14 \pm 238.49$ vs. 947.51 $\pm 298.42, \mathrm{P}<0.05$ ) (Table2).

Table 2: Leukocyte counts and cytokine concentration between groups.

\begin{tabular}{|c|c|c|}
\hline & IMN patients $(\mathbf{n}=24)$ & healthy control (n=12) \\
\hline IL-17 $(\mathrm{pg} / \mathrm{ml})$ & $56.02 \pm 9.54^{*}$ & $43.6 \pm 8.81$ \\
\hline TNF- $\alpha(\mathrm{pg} / \mathrm{ml})$ & $149.8 \pm 13.09^{*}$ & $36.51 \pm 7.79$ \\
\hline TGF- $\beta(\mathrm{pg} / \mathrm{ml})$ & $678.14 \pm 238.49^{*}$ & $947.51 \pm 298.42$ \\
\hline Th17\% & $1.15 \pm 0.62^{*}$ & $0.77 \pm 0.22$ \\
\hline Treg\% & $0.96 \pm 0.59^{*}$ & $1.67 \pm 0.52$ \\
\hline Th17/Treg & $1.15 \pm 0.33^{*}$ & $0.41 \pm 0.18$ \\
\hline
\end{tabular}

$* \mathrm{P}<0.05$ vs. heathy control 


\section{Relationship between Stratified Analysis of the Risk of IMN and Peripheral Th17 and Treg Cell Levels}

According to the 24-hour urinary protein level, all IMN patients were divided into middle- and high-risk groups. As illustrated in Table 3, the peripheral Th17\% and Th17/Treg ratio in the middlerisk groups were significantly up-regulated while the peripheral Treg\% was considerably down-regulated compared with normal control group (all $\mathrm{P}<0.05$ ), especially evident decline was noted in the high-risk group (all $\mathrm{P}<0.05$ ).

Table 3: Peripheral Th17 and Treg cell levels and cytokine concentration in patients with different risk of IMN.

\begin{tabular}{|c|c|c|c|}
\hline & $\begin{array}{c}\text { middle risk } \\
(\mathbf{n = 9})\end{array}$ & $\begin{array}{c}\text { high risk } \\
(\mathbf{n = 1 5})\end{array}$ & $\begin{array}{c}\text { healthy } \\
\text { control } \\
(\mathbf{n = 1 2})\end{array}$ \\
\hline $\mathrm{IL}-17(\mathrm{pg} / \mathrm{ml})$ & $52.78 \pm 7.54^{*}$ & $59.33 \pm 9.22^{*} \#$ & $43.6 \pm 8.81$ \\
\hline TNF- $\alpha(\mathrm{pg} / \mathrm{ml})$ & $149.8 \pm 13.09^{*}$ & $155.69 \pm 17.29^{*} \#$ & $36.51 \pm 7.79$ \\
\hline TGF- $\beta(\mathrm{pg} / \mathrm{ml})$ & $692.21 \pm 248.28^{*}$ & $574.22 \pm 197.15^{*} \#$ & $947.51 \pm 298.42$ \\
\hline Th17\% & $0.96 \pm 0.54^{*}$ & $1.82 \pm 0.60^{*} \#$ & $0.77 \pm 0.22$ \\
\hline Treg\% & $1.33 \pm 0.46^{*}$ & $1.12 \pm 0.41^{*} \#$ & $1.67 \pm 0.52$ \\
\hline Th17/Treg & $1.75 \pm 0.62^{*}$ & $0.77 \pm 0.54^{*} \#$ & $0.41 \pm 0.18$ \\
\hline
\end{tabular}

${ }^{*} \mathrm{P}<0.05$ vs. healthy control, $\# \mathrm{P}<0.05$ vs. middle risk.

Relationship between 24-hour Urinary Protein Level and Peripheral Th17 and Treg Cell Levels in IMN Patients

Correlation analysis between 24-hour urinary protein level and Th17 (Figure 1), Treg (Figure 2) and Th17/Treg ratio (Figure 3). As illustrated in Figure 4, the 24-hour urinary protein level was positively correlated with peripheral Th17\% and Th17/Treg ratio, and negatively correlated with peripheral Treg\% in IMN patients (all $\mathrm{P}<0.05$ ).

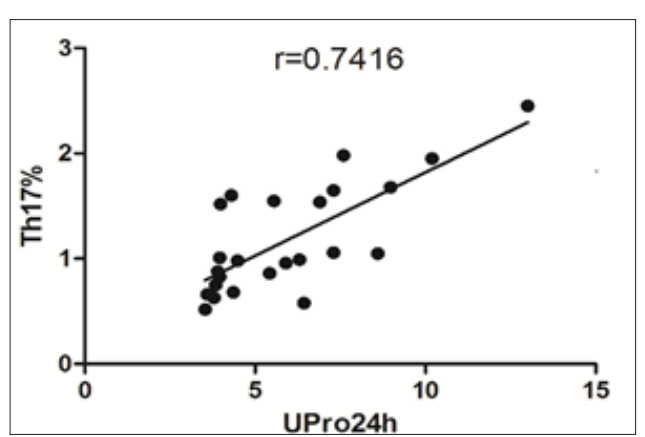

Figure 1.

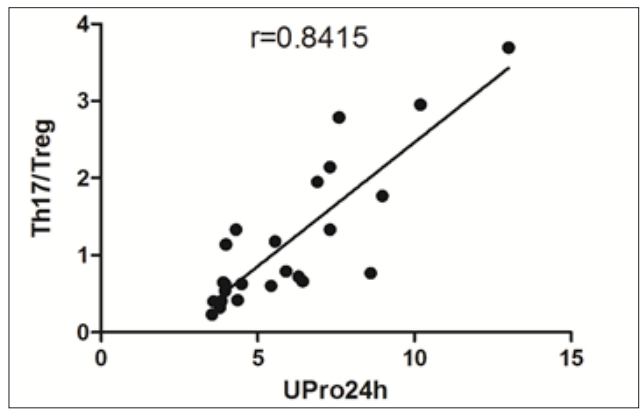

Figure 2.
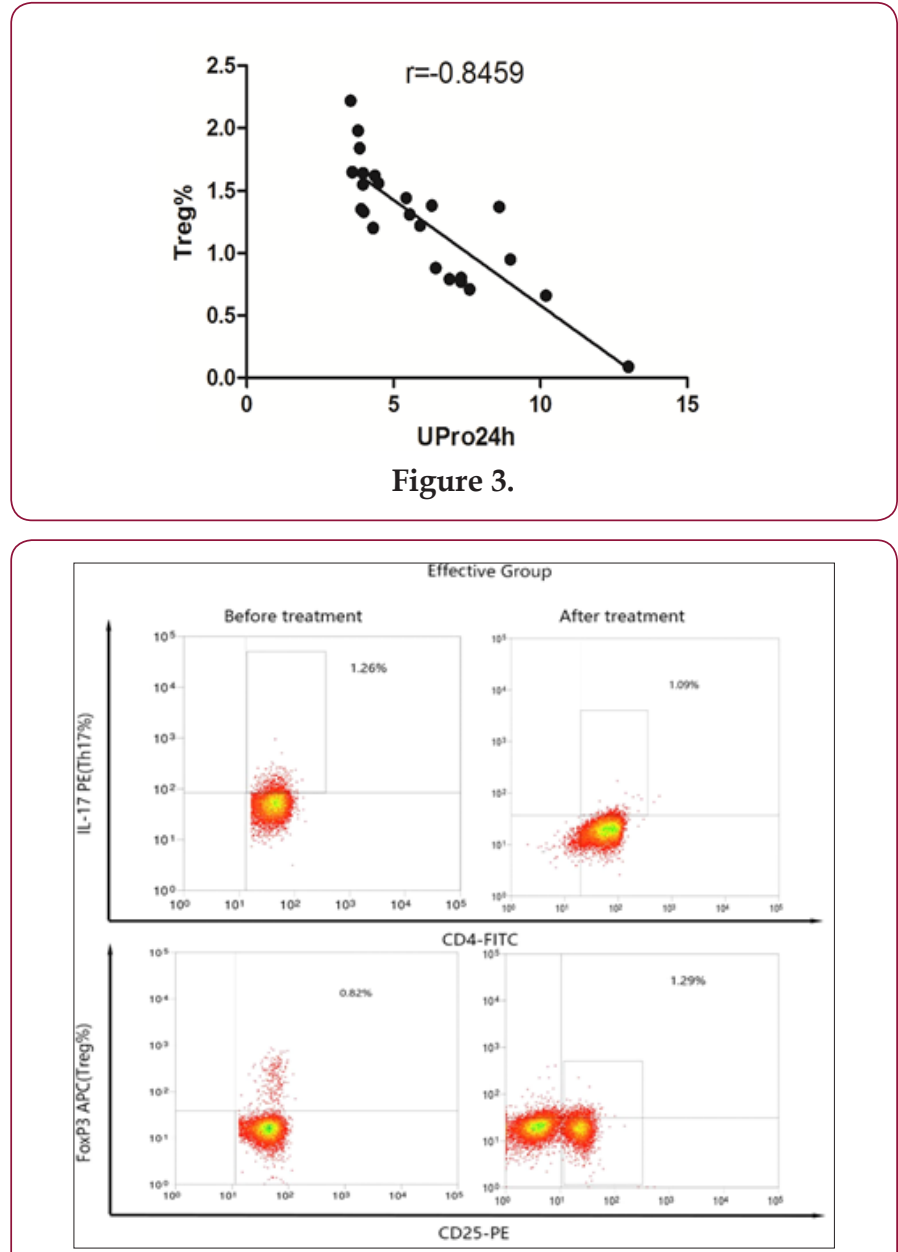

Figure 4: Correlation analysis between 24-hour urinary protein level and Th17 (Figure A), Treg (Figure B) and Th17/Treg ratio (Figure C).

Changes in clinical parameters and peripheral Th17, Treg and Th17/Treg ratio in IMN patients before and after CsA treatment

As illustrated in Table 4, the 24-hour urinary protein level, peripheral Th17\%, IL-17 and TNF- $\alpha$ levels were significantly downregulated, whereas the serum albumin level,peripheral Treg\% and TGF- $\beta$ level were remarkably up-regulated in IMN patients after CsA therapy for 6 months (all $\mathrm{P}<0.05$ ).

Table 4: Changes in clinical parameters and peripheral Th17, Treg and relevant cytokines in IMN patients after CsA treatment.

\begin{tabular}{|c|c|c|}
\hline & $\begin{array}{c}\text { IMN patients before } \\
\text { CsA treatment } \\
\text { (n=24) }\end{array}$ & $\begin{array}{c}\text { IMN patients after } \\
\text { CsA treatment } \\
\text { (n=24) }\end{array}$ \\
\hline 24 Upro(g) & $6.82 \pm 3.89^{*}$ & $4.36 \pm 2.35$ \\
\hline Alb(g/L) & $25.44 \pm 4.91^{*}$ & $32.46 \pm 3.24$ \\
\hline IL-17(pg/ml) & $56.02 \pm 9.54^{*}$ & $44.33 \pm 6.88$ \\
\hline TNF- $\alpha(\mathrm{pg} / \mathrm{ml})$ & $149.8 \pm 13.09^{*}$ & $99.14 \pm 10.3$ \\
\hline $\mathrm{TGF}-\beta 1(\mathrm{pg} / \mathrm{ml})$ & $678.14 \pm 238.49^{*}$ & $792.88 \pm 230.18$ \\
\hline $\mathrm{Th} 17 \%$ & $1.15 \pm 0.33^{*}$ & $0.92 \pm 0.84$ \\
\hline Treg\% & $1.26 \pm 0.48^{*}$ & $1.55 \pm 0.41$ \\
\hline Th17/Treg & $1.15 \pm 0.33^{*}$ & $0.69 \pm 0.36$ \\
\hline
\end{tabular}


${ }^{*} \mathrm{P}<0.05$ vs. before treatment\#P $<0.05$ vs. after treatment.

Changes in Clinical Parameters and Peripheral Th17, Treg and Relevant Cytokines in Sub-Group of IMN Patients After CsA Treatment

patients were assigned into the effective and ineffective groups according to the serum levels of Alb and 24-hour urinary protein. As illustrated in Table 5, In the effective group, the 24-hour urinary

Table 5: Changes in clinical parameters and peripheral Th17, Treg and relevant cytokines in sub-group of IMN patients after CsA treatment.

\begin{tabular}{|c|c|c|c|c|}
\hline & \multicolumn{2}{|c|}{ Effective Group $(\mathbf{n = 1 8})$} & \multicolumn{2}{c|}{ Ineffective Group (n=6) } \\
\hline & Before treatment & After treatment & Before treatment & After treatment \\
\hline 24-hour urinary protein(g/24h) & $6.82 \pm 3.89 \#$ & $4.64 \pm 2.63^{*} \#$ & $9.06 \pm 2.35 \#$ & $8.75 \pm 3.35 \#$ \\
\hline Alb(g/L) & $29.44 \pm 4.91 \#$ & $32.21 \pm 6.50^{*}$ & $23.44 \pm 3.24 \#$ & $24.48 \pm 4.09 \#$ \\
\hline IL-17(pg/ml) & $52.90 \pm 10.4 \#$ & $43.15 \pm 7.34^{*}$ & $63.43 \pm 8.65 \#$ & $61.63 \pm 12.08 \#$ \\
\hline TNF- $\alpha$ (pg/ml) & $123.8 \pm 7.91 \#$ & $81 \pm 6.5^{*}$ & $179.5 \pm 12.6$ & $175 \pm 5.14 \#$ \\
\hline TGF- $\beta$ (pg/ml) & $869.15 \pm 369.21 \#$ & $654.21 \pm 158.23^{*} \#$ & $1032.98 \pm 542.60 \#$ & $986.21 \pm 593.77 \#$ \\
\hline Th17\% & $1.05 \pm 0.54 \#$ & $0.81 \pm 0.43^{*}$ & $1.38 \pm 0.68 \#$ & $1.36 \pm 0.51 \#$ \\
\hline Treg\% & $1.11 \pm 0.60 \#$ & $1.64 \pm 0.39^{*}$ & $0.88 \pm 0.39 \#$ & $0.91 \pm 0.41 \#$ \\
\hline Th17/Treg & $0.99 \pm 0.48 \#$ & $0.45 \pm 0.22^{*}$ & $1.57 \pm 0.44 \#$ & $1.47 \pm 0.37 \#$ \\
\hline
\end{tabular}

$* \mathrm{P}<0.05$ vs. before treatment; $\# \mathrm{P}<0.05$ vs. after treatment.

Figure 5 Typical flow cytometry diagrams of peripheral Th7 and Treg cell levels in IMN patients before and after CsA treatment

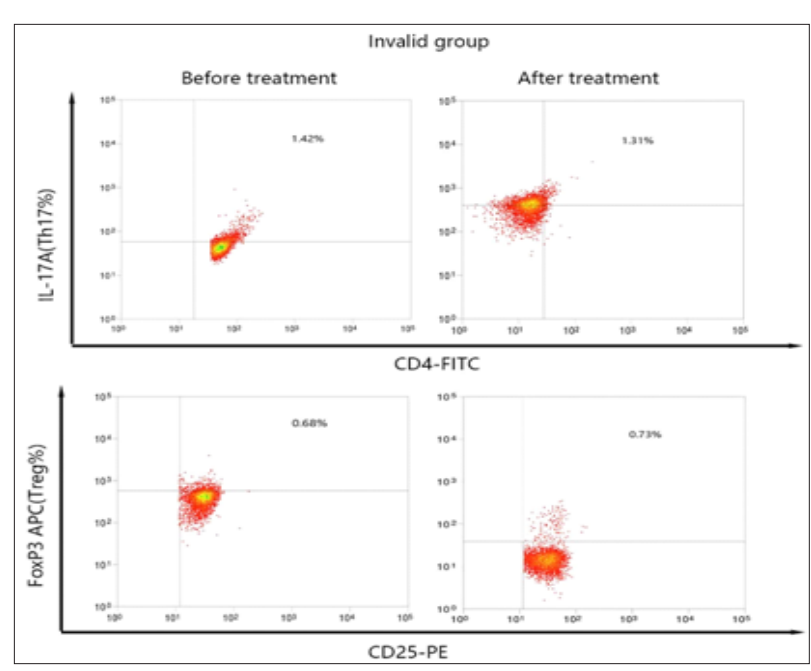

Figure 5: Typical flow cytometry diagrams of peripheral Th7 and Treg cell levels in IMN patients before and after CsA treatment.

\section{Discussion}

At present, the pathogenesis of idiopathic membranous nephropathy (IMN) is that the circulating autoantibody recognizes the target antigen of glomerular podocytes. After the antigenantibody binding, an immune complex forms subepithelially, which activates the complement to form membrane-attack complex, induces basement membrane and glomerular filtration barrier injury and generates albuminuria [9]. Until now, no biomarker has been applied to monitor the immune activity in IMN patients in clinical practice. Recent investigations have demonstrated that protein level, peripheral Th17\%, IL-17 and TNF- $\alpha$ levels were significantly down-regulated, whereas the serum level of Alb, peripheral Treg\% and TGF- $\beta$ were considerably up-regulated after CsA treatment (all $\mathrm{P}<0.05$ ). In the ineffective group, the 24-hour urinary protein level, serum level of Alb, peripheral Th17\%, Treg\%, IL-17, TNF- $\alpha$ and TGF- $\beta$ levels did not significantly differ before and after CsA treatment (all $\mathrm{P}>0.05$ ). 
and the secretion of cytokines (mainly IL-2). Besides, Treg can inhibit the activation of Th cells by antigen-presenting cells, suppress the activation of $\mathrm{B}$ cells by $\mathrm{Th}$ and represss the production of autoantibody by B cells, thereby mitigating the immune complexmediated injury to the tissues [13]. Thus, downregulation of Treg cells may active immune system and promote pathological reaction in the pathogenesis of IMN. In recent years, Th17 has been identified as a vital CD4+T cell subset. Th17 cells can specifically express nuclear transcription factor ROR $\gamma \mathrm{t}$, secrete IL-17, TNF- $\alpha$, IL-6 and IL-23, and participate in inflammatory response and tissue injury [14].

Cytokines secreted by Th17 cells can collectively mobilize, gather and activate neutrophils and mediate inflammatory reactions. Appropriate reactions can prevent tissue necrosis and pyemia, but it may cause tissue destruction and immune dysfunction if the response is too strong. therefore, the relative balance of Treg to Th17 is critical for maintening immune tolerance and preventing inflammatory lesions [15]. In this study, Th17 cell and the levels of IL-17, TNF- $\alpha$ and IL-6 were up-regulated in IMN patients, prompting that IMN initiates the Th17-type immune response probably through the release of IL-17, TNF- $\alpha$ and alternative proinflammatory cytokines, thereby provoking regional kidney tissue inflammation and up-regulating the expression of IL-6, MCP-1 and other pro-inflammatory cytokines and chemokines. Further subgroup analysis revealed that $\mathrm{Th} 17 \%$ and $\mathrm{Th} 17 /$ Treg ratio were higher while Treg\% was lower in midille risk group when compared to nomal control group, this change was most significant in high risk group.

A Treg/Th17 cell imbalance was found to correlate with 24hours total urine protein, and the patients with a greater Treg/Th17 imbalance had more 24hours total urine protein. We suggest that simultaneous Th17 cell upregulation and Treg cell downregulation may lead to progression of IMN through immunemediated injury, and a greater Treg/Th17 imbalance is associate with prognosis. Therefore, immunotherapy with the goal of decreasing the inflammations caused by Treg/Th17 imbalances may have a protective effect in patients with IMN. As a common drug for membranous nephropathy, CsA can be utilized as the initial treatment of IMN or alternative therapy if other medications are ineffective. The primary mechanism underlying the decrease of albuminuria is to inhibit immune response, selectively suppress the activation of $\mathrm{T}$ cells, repress the production of IL-2, inhibit the secretion of calcineurin, block the dephosphorylation of synaptopodin induced by calcineurin and stabilize the cytoskeleton of kidney podocyte, thereby reducing the generation of protein [16].

Previous studies $[17,18]$ have reported that administration of CsA exerts a significant effect upon immune diseases and after organ transplantation, which is possibly associated with clinical efficacy.

In this study, the variation in Th17/Treg ratio in IMN patients was observed before and after CsA treatment. In the effective group, the level of Th17 was significantly down-regulated, whereas the level of Treg was considerably up-regulated after CsA therapy, hinting that CsA probably affects clinical efficacy and prognosis of IMN patients by regulating the Th17/Treg immune imbalance. Moreover, Th17/Treg ratio did not significantly alter after CsA therapy in the ineffective group, prompting that Th17/Treg ratio can be used to evaluate the severity of IMN and clinical prognosis. Taken together, IMN patients present with peripheral Th17/Treg imbalance, which is correlated with the severity of IMN. CsA therapy is an efficacious approach to improve the peripheral Th17/Treg imbalance, which is linked to the clinical efficacy of CsA treatment. Dynamic monitoring of the variation in the peripheral levels of Treg and Th17 contributes to evaluate the severity of IMN and assess the clinical efficacy.

\section{Acknowledgment}

The authors thank Mrs Liu Juan for experimental support.

\section{References}

1. Kwatra IS, Prasher PK (2013) Pathogenesis of membranous nephropathy: update. J Assoc Physicians India 61(11): 807-810.

2. Shi X, Qu Z, Zhang L, Zhang N, Liu Y, et al. (2016) Increased ratio of ICOS (+) /PD-1(+) follicular helper T cells positively correlates with the development of human idiopathic membranous nephropathy. Clin Exp Pharmacol Physiol 43(4): 410-416.

3. Schmitt V, Rink L, Uciechowski P (2013) The Th17/Treg balance is disturbed during aging[J]. Experimental Gerontology 48(12): 13791386.

4. Jia XY, Hu SY, Chen JL, Qu Z, Liu G, et al. (2014) The clinical and immunological features of patients with combined anti-glomerular basement membrane disease and membranous nephropathy. Kidney International: Official Journal of the International Society of Nephrology 85(4): 945-952.

5. Iannitti RG, Carvalho A, Cunha C, De Luca A, Giovannini G, et al. (2013) Th17/Treg imbalance in murine cystic fibrosis is linked to indoleamine 2,3-dioxygenase deficiency but corrected by kynurenines. American journal of respiratory and critical care medicine 187(6): 609-620.

6. Rosenzwajg M, Languille E, Debiec H, Hygino J, Dahan K, et al. (2017) $\mathrm{B}$-and T-cell subpopulations in patients with severe idiopathic membranous nephropathy may predict an early response to rituximab. Kidney Int 92(1): 227-237.

7. Tang B, Ren H, Liu H, Shi Y, Liu W, et al. (2016) CCR5 blockade combined with cyclosporine A attenuates liver GVHD by impairing T cells function. Inflamm Res 65(11): 917-924.

8. Sakai R, Taguri M, Oshima K, Mori T, Ago H, et al. (2016) A comparison of tacrolimus and cyclosporine combined with methotrexate for graftversus-host disease prophylaxis, stratified by stem cell source: a retrospective nationwide survey. Int J Hematol 103(3): 322-333.

9. Mercadal L (2013) Membranous nephropathy. Nephrol Ther 9: 507-17.

10. Wangbo, Liu Zhi-hong, WU Yan, Zuo Ke, Huangq ian, et al. (2009) RegulatoryT cells and B cells in patients with idiopathic membranous nephropathy. Chinese Journal of Nephrology, Dialysis \& Transplantation 18: 322-28.

11. Masutani K, Taniguchi M, Nakashima H, Yotsueda H, Kudoh $\mathrm{Y}$, et al. (2004) Up-regulated interleukin-4 production by peripheral T-helper cells in idiopathic membranous nephropathy. Nephrol Dial Transplant 19: 580-586.

12. Zhang J, Hua G, Zhang X, Tong R, Du X, et al. (2010) Regulatory T cells/Thelper cell 17 functional imbalance in uraemic patients on maintenance haemodialysis: a pivotal link between microinflammation and adverse cardiovascular events. Nephrology (Carlton) 15: 33-41 
13. Kim BS, Nishikii H, Baker J, Pierini A, Schneidawind D, et al. (2015) Treatment with agonistic DR3 antibody results in expansion of donor Tregs and reduced graft-versus-host disease. Blood 126: 546-557.

14. Wang L, Li Q, Wang L, Li C, Yang H, et al. (2013) The role of Th17/IL-17 in the pathogenesis of primary nephrotic syndrome in children. Kidney Blood Press Res 37: 332-345.

15. Liu LL, Qin Y, Cai JF, Wang HY, Tao JL, et al. (2011) Th17/Treg imbalance in adult patients with minimal change nephrotic syndrome. Clin Immunol 139: 314-320.

\section{ISSN: 2574-1241}

DOI: 10.26717/BJSTR.2018.07.001579

JianBin Zhang. Biomed J Sci \& Tech Res

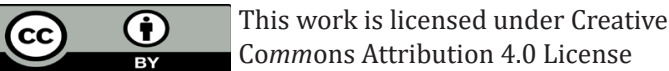

Submission Link: https://biomedres.us/submit-manuscript.php
16. Foxwell BM, Ruffel B (1990) The mechanisms of action of cyclosporine. Cardiol Clin 8: 107-117.

17. Jaiswal A, Prasad N, Agarwal V, Yadav B, Tripathy D, et al. (2014) Regulatory and effector $\mathrm{T}$ cells changes in remission and resistant state of childhood nephrotic syndrome. Indian J Nephrol 24(6): 349-355.

18. Hunemorder S, Treder J, Ahrens S, Schumacher V, Paust HJ, et al. (2015) TH1 and TH17 cells promote crescent formation in experimental autoimmune glomerulonephritis. J Pathol 237(1): 62-71.

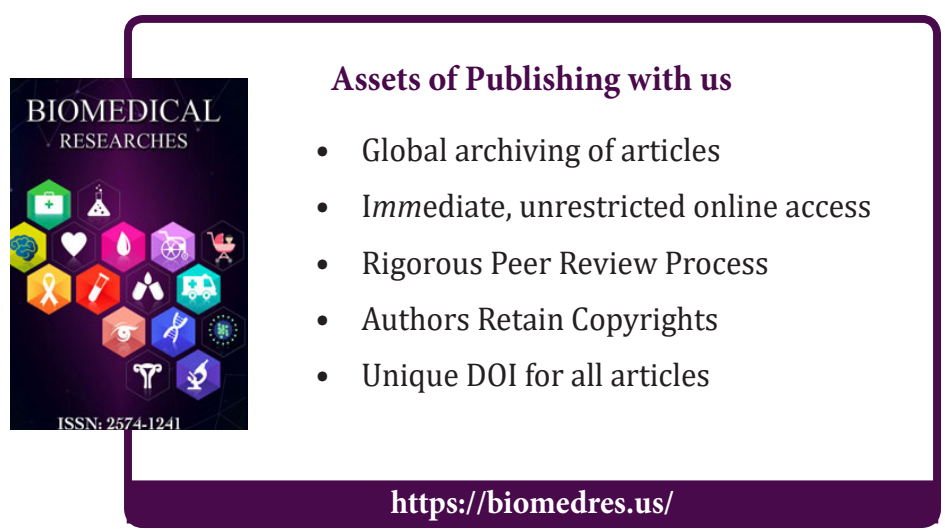

